\section{Long-term follow-up of true hermaphroditism cases treated by conservative surgery}

Verkauskas and colleagues have retrospectively reviewed data from their 40-year study on true hermaphroditism-now called ovotesticular disorder of development-a rare condition in which individuals have both testicular and ovarian tissue.

Children ( $n=33)$ with true hermaphroditism were followed up for a median of 11 years; 5 were diagnosed prenatally and 28 postnatally. Most patients (82\%) had a $46, \mathrm{XX}$ karyotype; $35 \%$ of these had the SRY gene (sex-determining region $Y$, which encodes testis-determining factor). All patients underwent conservative surgery, which aims to preserve all gonadal tissue concordant with sex of rearing ( 21 as female and 12 as male). The most common gonadal structure before surgery was the ovotestis (in $65 \%$ of patients), and testis was the rarest finding (9\%). Right-side ovotestes consistently contained more testicular tissue than left-side ovotestes. Consistent with previous reports, ovarian tissue remained functional until $\geq 17$ years of age, whereas testicular dysgenesis occurred over time. Testosterone production, however, remained at a satisfactory level in most male-reared individuals during follow-up. Of consequence, there were no fertile individuals in this cohort.

Early diagnosis of true hermaphroditism is now possible. The authors provide an algorithm for prenatal detection of this condition and suggest that in many cases it is preferable to raise affected children as girls, since their ovarian tissue remains functional. The study highlights the considerable psychological, gynecological and urological problems of individuals in this series, and stresses the need for long-term follow-up.

Original article Verkauskas G et al. (2007) The long-term followup of 33 cases of true hermaphroditism: a 40-year experience with conservative gonadal surgery. J Urol 177: 726-731

\section{Laparoscopic and open radical prostatectomy have equivalent urinary outcomes}

Laparoscopic and open radical prostatectomy have similar perioperative outcomes, but the lack of consistency in definitions of postoperative continence has resulted in wide ranges of reported postoperative continence rates for these techniques. The few published studies that compared these procedures directly used subjective measures of continence.

Jacobsen and colleagues prospectively compared postoperative continence rates and urinary symptoms in men with clinically localized prostate cancer who underwent either open radical retropubic prostatectomy (RRP, $n=148$ ) or laparoscopic radical prostatectomy (LRP, $n=57$ ) at a single hospital. Preoperative evaluations included a $24 \mathrm{~h}$ pad test, completion of a fluid intake and voiding diary, and the International Prostate Symptom Score (IPSS). During 12 months' postoperative follow-up, the IPSS was repeated every 3 months, and the pad test and diary assessments were repeated at 3 and 12 months. Interestingly, although all patients subjectively reported complete preoperative continence, $4 \%$ of men in the RRP group and $3.5 \%$ of men in the LRP group were objectively incontinent ( $24 \mathrm{~h}$ pad weight gain $\geq 8 \mathrm{~g}$ ). Incontinence rates peaked at 3 months postoperatively in both groups $(66.7 \%$ in the RRP group and $41.3 \%$ in the LRP group). By 12 months, however, there were no differences in postoperative continence rates, IPSSs, or urinary quality of life between the groups.

Jacobsen and colleagues note that their finding of objectively and subjectively equivalent 12-month postoperative urinary and functional outcomes for RRP and LRP is not unexpected, given that LRP aims to duplicate the continence-preserving features of RRP.

Original article Jacobsen N-EB et al. (2007) Open versus laparoscopic radical prostatectomy: a prospective comparison of postoperative urinary incontinence rates. J Urol 177: 615-619

\section{Serum biomarker JM-27 could distinguish symptomatic from asymptomatic BPH}

Benign prostatic hyperplasia $(\mathrm{BPH})$ has traditionally been considered a single disease entity, but genomic studies revealed different geneexpression profiles in individuals with symptomatic and asymptomatic disease. These studies identified JM27, an androgen-regulated gene expressed in normal prostate and testis, strongly expressed in asymptomatic $\mathrm{BPH}$, and very strongly expressed in symptomatic $\mathrm{BPH}$. 